\title{
An Optimal Adaptive M-PSK Carrier Phase Detector Suitable for Fixed-Point Hardware Implementation within FPGAs and ASICs
}

\author{
Yair Linn, Member IEEE \\ University of British Columbia \\ 6335 Thunderbird Crescent, Box 341, Vancouver, BC, Canada V6T-2G9 \\ e-mail: ylinn@ece.ubc.ca
}

\begin{abstract}
In this paper we shall present an NDA (Non Data Aided) adaptive carrier phase detector for coherent M-PSK receivers operating in AWGN (Additive White Gaussian Noise). It shall be shown that the detector allows the carrier synchronization PLL (Phase Locked Loop) to achieve optimal performance during both the acquisition and tracking operation modes. The conditions necessary for this optimality to be achieved will be discussed, and it shall be shown that they are quite reasonable and allow the proposed detector to be implemented in many contemporary M-PSK receivers. The optimal behaviour of the PLL will be shown to hold regardless of the SNR (Signal-to-Noise Ratio) and of the AGC (Automatic Gain Control) circuit behaviour. Moreover, the proposed detector has a simple fixed-point structure that can be feasibly implemented using few hardware resources within contemporary FPGAs (Field Programmable Gate Arrays) or ASICs (Application Specific Integrated Circuits). Finally, operation of the proposed detector under frequency-flat slow signal fading conditions is also discussed.
\end{abstract}

\section{INTRODUCTION}

In coherent M-PSK receivers that use a carrier synchronization PLL for coherent demodulation, these PLLs attempt to cancel the phase error between the received signal's carrier and the local oscillator. The PLL operates on an estimate of the phase error, which is provided by a carrier Phase Detector (PD). PDs can be classified into two general subcategories: Non Data Aided (NDA) and Decision Directed (DD). Some examples of NDA detectors are the $\mathrm{M}^{\text {th }}$-order nonlinearity detector ([1 Chap. 6], [2 Chap. 5, 6], [3 Chap. 5]) and the multiphase NDA Costas loop ([4], [3 Chap. 5]), and DD detectors are discussed in [1 Chap. 6], [2 Chap. 5, 6], [3 Chap. 5], [5], [6], [7] and [8].

In [9] it was shown that the NDA and DD detectors just cited suffer from two major drawbacks. First, at low SNRs and during acquisition they suffer from considerable self-noise, which causes a performance degradation. Secondly, their gain is strongly dependent both upon (a) the SNR and (b) the AGC circuit's operating point and performance. As shown in [9 Sec. 7], the fact that the gain of the phase detector is not constant implies that the carrier PLL's parameters will exhibit corresponding variations. In order to combat the aforementioned shortcomings, Linn suggested in [9 Sec. 5] a simple adaptive phase detector structure that allows the PLL to perform optimally at virtually any SNR and almost independent of AGC performance. While clearly superior to previously available PDs, the structure in [9] only provides for optimal behaviour of the carrier PLL when the latter is locked, i.e. when the PLL is tracking. During acquisition, the structure of [9] only provides optimal performance at a single SNR.

The purpose of this paper is to introduce an NDA adaptive phase detector structure for M-PSK carrier synchronization which allows the carrier PLL to perform optimally during both acquisition and tracking, at all feasible SNRs, nearly independently of the AGC's behaviour. The proposed structure is essentially an elaboration upon the structure discussed in [9], where the modification of the latter is such that optimal performance is also achieved during acquisition.

This paper draws heavily upon [9] and [10]. The reader is thus encouraged to take a look [9] and [10] before continuing, since these papers contain many derivations and definitions that shall not be repeated here due to space constraints.

\section{System Model}

We define the baseband signal as $m(t) \triangleq \sum_{n=-\infty}^{\infty} a_{n} p(t-n T)$, with $p(t)$ being the baseband data pulse with energy $E_{p} \triangleq \int_{-\infty}^{\infty} p^{2}(t) d t$, and $a_{n}=\exp \left(j \phi_{n}\right), \phi_{n}=2 \pi \cdot m_{n} / M$, with $m_{n} \in\{0,1, \ldots, M-1\}$. The modulated signal is $s_{m}(t) \triangleq \operatorname{Re}\left[m(t) \exp \left(j \omega_{i} t+j \theta_{i}\right)\right]$ and that signal is corrupted by AWGN. Fig. 1 shows the M-PSK receiver, where:

1) $1 / T$ is the both the symbol rate and the sample rate.

2) $n(t) \sim N\left(0, N_{0} W\right)$ where $W$ is the width of the bandpass IF filter (not shown).

3) $K$ represents circuit's AGC-controlled equivalent gain. For a detailed discussion of the AGC and $K$ see [9 App. A].

4) When the carrier PLL is locked, we have $\Delta \omega=0$ and (since M-PSK carrier synchronization has an M-fold ambiguity [3 Sec. 5.7.6]) $\quad \theta_{o} \in\left\{\theta_{i}+2 \pi k / M-\theta_{e} \mid k=0,1, \ldots, M-1\right\}, \quad$ where $\theta_{e} \in[-\pi / M, \pi / M]$ is the residual phase error; without loss of generality, we assume $k=0$ in the remainder of this paper. Conversely, when unlocked, $\Delta \omega \neq 0$ and $\theta_{e} \triangleq\left(\theta_{i}-\theta_{o}\right) \bmod _{2 \pi} \in[-\pi, \pi]$.

5) The matched filter $h(t)$ is assumed ideal and the sampling at the outputs of matched filters is considered to be at the ideal time (i.e. the symbol synchronization loop is assumed locked).

6) From [1 Sec. 4.1.1] the symbol energy is $E_{S}=1 / 2 E_{p}$. For convenience we assume $E_{P}=1$ (implying $E_{S}=1 / 2$ ), and we use the notation $\chi$ to refer to the $E_{S} / N_{0}$ ratio (=SNR).

7) We assume a narrowband signal (i.e. $\omega_{i}>>1 / T$ ) for which the Nyquist criterion for zero-ISI [1 Sec. 9.2.1] is obeyed regarding the output of the matched filters.

8) We make the standard assumption ([2], [3]) that the carrier PLL is a high-loop-gain second-order system. The linearizedmodel Laplace transfer function of the PLL is thus $H_{P L L}(s) \triangleq \theta_{o}(s) / \theta_{i}(s)=\frac{2 \zeta \omega_{n} \cdot s+\omega_{n}^{2}}{s^{2}+2 \zeta \omega_{n} \cdot s+\omega_{n}^{2}}$ where $\zeta$ is the damping ratio and $\omega_{n}$ (radians/sec) is the natural frequency.

\section{Modeling of Phase Detectors - a Brief Overview}

Theoretical modeling of phase detector operation is customarily done through the PLL's linear model. Thus, in order for us to pursue the analysis of the proposed phase detector, we must first define some linear-model variables which shall be used in that characterization. For any phase detector $P(n)$ we have the following characteristics:

1) $B_{L} \triangleq \int_{0}^{\infty}\left|H_{P L L}(j 2 \pi f)\right|^{2} d f=1 / 2 \omega_{n}(\zeta+1 /(4 \zeta))$ is the PLL's noise bandwidth (see [11 p. 30-32]).

2) The PD's $S$-Curve [3 p. 206] is $S_{P}\left(\theta_{e}\right) \triangleq E\left[P(n) \mid \theta_{e}\right]$

3) The gain of $P(n)$ is:

$\left.g_{P}(M, K, \chi) \triangleq\left(\partial S_{P}\left(\theta_{e}\right) / \partial \theta_{e}\right)\right|_{\theta_{e}=0}$ 
The gain is usually normalized so that it is unity at $E_{S} / N_{0}=\infty$. Most synchronization texts also assume a constant $K=1$, whereupon the normalized gain is:

$\alpha_{\chi, P} \triangleq g_{P}(M, 1, \chi) / g_{P}(M, 1, \infty)$

$\alpha_{\chi, P}$ is called the amplitude suppression factor. However as shown in [9 App. A], despite its prevalent use, the assumption $K=1$ is not realistic. Hence, in this paper we assume that $K$ is a function of the SNR, i.e. $K=\Upsilon_{A G C}(\chi)$ [9 App. A], and we define the effective amplitude suppression factor $\beta_{\chi, P}$, which is used for modeling AGC effects in the PLL[9]. Formally:

$\beta_{\chi, P} \triangleq g_{P}\left(M, \Upsilon_{A G C}(\chi), \chi\right) / g_{P}(M, 1, \infty)$

4) The PLL's normalized equivalent loop noise at $\theta_{e} \approx 0$ is (using [2 eq. (6-73), p. 342] and normalizing ${ }^{1}$ )

$N_{e, P}(n) \triangleq \lim _{\theta_{e} \rightarrow 0}\left(P(n)-S_{P}\left(\theta_{e}\right)\right) / g_{P}(M, 1, \infty)$.

5) The PD's self noise is ${ }^{1}$ [5 eq. (6)]

$\xi_{P} \triangleq 2 \cdot \chi \cdot \operatorname{var}\left(N_{e, P}(n)\right)$.

6) The PD's squaring loss [1 eq. (6.2-59)] is $\Omega_{P} \triangleq \xi_{P} / \alpha_{\chi, P}^{2}$ if we assume $K=1$, or $\Omega_{P} \triangleq \xi_{P} / \beta_{\chi, P}^{2}$ if modeling of the AGC's effects is done via $K=\Upsilon_{A G C}(\chi)$ ( $\Omega_{P}$ is identical in both cases ${ }^{1}$, since it is one of the PD's inherent properties).

7) Finally, the PLL's phase-error variance is ${ }^{2}$ [6 eq. (21)] : $\operatorname{var}\left(\theta_{e}\right)=B_{L} \cdot T \cdot \Omega_{P} / \chi=1 / 2 \omega_{n}(\zeta+1 /(4 \zeta)) \cdot T \cdot \Omega_{P} / \chi$ (6)

The linear model is shown in [9 Fig. 4, lower left], with $\alpha_{S N R}$ denoting $\alpha_{\chi, P}$. When AGC effects are modeled, then $\beta_{\chi, P}$ replaces $\alpha_{S N R}$ in that schematic (also see footnote 1).

\section{Motivation ANd Detector Structure}

In this section we assume (for simplicity and in order to understand the proposed algorithm intuitively) that no fading is present. In Sec. V we extend the analysis to include fading effects.

\section{A. Motivation}

In [9 Sec. 7.1] it is shown that a phase detector needs to have an effective amplitude suppression factor of 1 at all SNRs in order for the PLL to have a constant loop gain at all SNRs. This in turn allows the PLL's parameters to remain constant and hence (if chosen correctly) optimal ${ }^{3}$ at all SNRs. We can easily see how to transform any given phase detector $P(n)$ into a phase detector

$R(n)$ for which $\forall \chi, \beta_{\chi, R}=1$, both during tracking and acquisition, using the schematic of Fig. 4 . Note that in Block \#6 there is division of $P(n)$ by $g_{P}(M, 1, \infty) \cdot \hat{\beta}_{\chi, P}$, which ensures not only that $\forall \chi, \beta_{\chi, R}=1$, but also (from (3)) that $\forall \chi$, $g_{R}(M, K, \chi)=1$ (i.e. that $R(n)$ has unity gain at all SNR) which further simplifies the PLL's analysis and implementation. Fig. 4 is the general idea of the adaptive detector presented in this paper, which is now outlined.

\section{B. Detector Structure}

The structure of $U_{M, N^{C}, N^{D}}$ is shown in Fig. 5. Formally:

\footnotetext{
${ }^{1}$ When AGC effects are ignored then (4) and (5) are computed with $K=1$ assumed. When AGC effects are modeled and $\beta_{\chi, P}$ is used in the linear model, then (4) and (5) are computed with $K=\Upsilon_{A G C}(\chi)$.

${ }^{2}$ Note that $B_{L}$ here contains a factor of $1 / 2$ w.r.t. its definition in [6], but (6) compensates with a factor of 2 w.r.t. [6 eq. (21)].

${ }^{3}$ Sometimes, the optimal value of $\omega_{n}$ will be SNR dependent [12 Chaps. 7, 8] However, even in that case the desired variation of $\omega_{n}$ will not usually corresponds to the variation induced by the changing phase detector gain, so we would rather have a constant-gain PD and modify $\omega_{n}$ via changing the loop filter coefficients.
}

$U_{M, N^{C}, N^{D}}(n) \triangleq \begin{cases}(1 / M) \cdot\left(d_{M}(n) / \hat{\lambda}^{C}\right) & \text { Carrier loop is locked } \\ (1 / M) \cdot\left(d_{M}(n) / \hat{\lambda}^{D}\right) & \text { Carrier loop is unlocked }\end{cases}$

where $d_{M}(n) \triangleq \operatorname{Im}\left[(I(n)+j Q(n))^{M}\right] /\left(I^{2}(n)+Q^{2}(n)\right)^{M / 2}$ is the normalized $\mathrm{M}^{\text {th }}$-order nonlinearity detector (see [9 Sec. 4]).

The correspondence between the modules of Fig. 5 and the modules of Fig. 4 can be clearly seen through the labels Block \#1 through Block \#6 in both figures. Essentially, Fig. 5 is the implementation of Fig. 4 for $d_{M}(n)$.

We note and stress and that though the implementation of Fig. 5 may seem complicated at first glance, as we shall now show it is in fact very easy to implement in hardware.

It is instructive to look separately above and below the dashed line that bisects Fig. 5. First, let us look at the lower part. Below the dashed line, the schematic bears an uncanny resemblance to the Linn adaptive phase detector shown in [9 Fig. 2]. This is no coincidence: indeed, if one follows Fig. 5 for the case of when the carrier loop is locked, it is easy to see that $U_{M, N^{C}, N^{D}}$ is derived

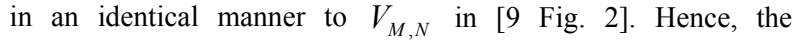
behaviour of $U_{M, N^{C}, N^{D}}$ during tracking is identical to that of $V_{M, N}$ (with $N=N^{C}$ ), as analyzed in [9]. Thus, since $V_{M, N}$ had $\forall \chi, \beta_{\chi, V}=1$ during tracking [9 Secs. 6, 7], then $\forall \chi, \beta_{\chi, U}=1$ during tracking. With regards to fixed-point hardware implementation, recall that in [9 Sec. 5.2] it is shown that $V_{M, N}$ 's implementation is compact, so that this also applies to the lower half of Fig. 5. Furthermore, in [9] it is shown that $V_{M, N}$ is resilient to imperfection in the AGC circuit, a conclusion that now also applies to $U_{M, N^{C}, N^{D}}$ during tracking.

Now, let us assume that the carrier is unlocked and let's look at the schematic of Fig. 5 above the bisecting dashed line. We observe an uncanny resemblance to another structure, this time to the D-MPSK SNR estimation structure shown in [10 Fig. 2]. Again, this resemblance is no coincidence. The are two difference, however, in Fig. 5: the contents of LUT D and the existence of MUX1.

Paper [10] presented a structure for SNR estimation for DMPSK based on the Linn-Peleg lock detector and SNR estimator ([13], [14]). As noted in [10 Sec. VIII], the estimator in [10] can also be used for SNR estimation for M-PSK in the absence of carrier synchronization, and that is how we employ it here.

In [10 Fig. 2] we treated SNR estimation for D-MPSK, and therefore the output of that structure was an SNR estimate. Here, we are trying to estimate $\beta_{\chi, d}$, and, therefore, LUT D performs the transformation $\hat{l}_{M, N^{D}}^{D} \rightarrow \beta_{\chi, d}$. LUT D will be discussed in further detail in Sec VII.

As for MUX1, while we could theoretically use $d_{M}(n) /\left(M \cdot \lambda^{D}\right)$ as a unity-gain phase detector in the case of carrier unlock, due to the ability of $\lambda^{D}$ to vanish this would imply that LUT G would need to accommodate unbounded outputs, hence hindering its implementation in fixed-point hardware. This is easily solved by the using $\hat{\lambda}^{D}$ instead of $\lambda^{D}$, where $\hat{\lambda}^{D}= \begin{cases}\lambda^{D} & \lambda^{D}>\lambda_{\text {Thr }}^{D} \\ \lambda_{\text {Default }}^{D} & \lambda^{D}<\lambda_{\text {Thr }}^{D}\end{cases}$ as shown in Fig. 5. The

choice of $\lambda_{T h r}^{D}$ is straightforward: we can choose $\lambda_{T h r}^{D}=\beta_{\Gamma, d}$ where $\Gamma$ is the lock threshold of the PLL (below $\Gamma$ the PLL cannot lock so its operation is of no interest to us). The case of $\lambda^{D}<\lambda_{\text {Thr }}^{D}$ corresponds to either (a) there is no input signal, (b) there is an input signal but its SNR is below the lock threshold, or 
(c) there is an input signal and its SNR is above the lock threshold but due to the variance of $\hat{l}_{M, N^{D}}^{D}$ we still have $\lambda^{D}<\lambda_{T h r}^{D}$. In all three cases this can be thought of as a situation where we lack information about the SNR of the input signal, and hence a logical choice for $\lambda_{\text {Default }}^{D}$ would be $\lambda_{\text {Default }}^{D}=\lambda_{\text {Thr }}^{D}$ since this would optimize the phase detector gain for operation at the lock threshold.

The same reasoning applies to the PLL during lock (see [9 Sec. 5.2]). We thus assume $\lambda_{\text {Default }}^{D}=\lambda_{\text {Thr }}^{D}=\lambda_{\text {Thr }}^{C}=\beta_{\Gamma, d}$, whereupon we see that the largest value that needs to be accommodated by LUT G in this case would be $d_{M}(n) /\left(M \cdot \beta_{\Gamma, d}\right)$. Now, $\beta_{\Gamma, d} \geq 0.04$ since below that value the SNR for the respective modulations is so low that lock is unachievable (see [9 Sec. 5.2], [13 Fig. 3]). Thus the largest absolute value that needs to be accommodated by LUT $\mathrm{G}$ would be $\quad \sup \left|d_{M}(n)\right| /\left(M \cdot \inf \left(\beta_{\Gamma, d}\right)\right)=1 /(M \cdot 0.04)=25 / M$ which means that the dynamic range of LUT G can be sufficiently limited to allow compact implementation in fixed-point hardware.

As for the other components of $U_{M, N^{C}, N^{D}}$ which appear above the dashed line, these were discussed in [10] where it was shown that they can be compactly implemented in fixed-point logic within an FPGA or ASIC. Moreover, since the structure in [10] is robust vis-à-vis imperfection in the AGC's operation, this is also true of $U_{M, N^{C}, N^{D}}$ during acquisition.

To conclude this section, we have outlined the structure of the new detector $U_{M, N^{C}, N^{D}}$ and we have shown that it can be implemented compactly in fixed-point hardware and that is it robust with regards to AGC circuit imperfections.

\section{V. $U_{M, N^{C}, N^{D}}$ IN The Presence of Fading}

The previous section assumed that no fading is present. In this section, we shall see how the analysis of the previous section can be extended to include such effects. In this paper, as in [10] (but unlike [9], [14], and [13]) we shall investigate the proposed phase detector in the presence of frequency-flat slow fading [1 Chap. 14]. To do this we first define the notation $\bar{\chi}$ as the average SNR, given by $\bar{\chi} \triangleq E\left[E_{S} / N_{0}\right]$. The conditional pdf (probability density function) of the SNR due to fading is defined as:

$p_{F}(\chi \mid \bar{\chi}) \triangleq p\left(E_{S} / N_{0}=\chi \mid E\left[E_{S} / N_{0}\right]=\bar{\chi}\right)$

For example, for Nakagami- $m$ fading [15 Table 2]

$p_{F}(\chi \mid \bar{\chi})=\frac{m^{m} \chi^{m-1}}{\bar{\chi}^{m} \Gamma(m)} \exp \left(\frac{-m \chi}{\bar{\chi}}\right)$

Just like in [10], we must pursue analysis of $U_{M, N^{C}, N^{D}}$ according to the channel coherence time $T_{\mathrm{COH}}$ [1 Sec. 14.1.1] When $2 N^{C} T \ll T_{\mathrm{COH}}$ or $2 N^{D} T \ll T_{\mathrm{COH}}$ then since the SNR will not have changed much during the computation interval then $\hat{\lambda}^{C}$ or $\hat{\lambda}^{D}$ will provide an estimate of the instantaneous effective amplitude suppression factor $\beta_{\chi, d}$ where $\chi$ is, we recall, the instantaneous SNR. When $2 N^{C} T \gg T_{\mathrm{COH}}$ or $2 N^{D} T \gg T_{\mathrm{COH}}$ then the fading distribution will have influenced $\hat{\lambda}^{C}$ or $\hat{\lambda}^{D}$ (respectively) so that they give an estimate of the average effective amplitude suppression factor, defined as :

$\bar{\beta}_{\bar{\chi}, d} \triangleq E\left[\beta_{\chi, d} \mid E\left[{ }^{E_{S}} / N_{0}=\bar{\chi}\right]\right]=\int_{0}^{\infty} \beta_{\chi, d} \cdot p_{F}(\chi \mid \bar{\chi}) d \chi$

Thus, we differentiate between 3 cases, as follows:

(a). $2 N^{C} T \ll T_{\mathrm{COH}}$ and $2 N^{D} T \ll T_{\mathrm{COH}}$

(b). $2 N^{C} T \ll T_{\mathrm{COH}}$ and $2 N^{D} T \gg T_{\mathrm{COH}}$ (c). $2 N^{C} T \gg T_{\mathrm{COH}}$ and $2 N^{D} T \gg T_{\mathrm{COH}}$

In this classification we make the assumption that $N^{D}>N^{C}$ (an assumption that will be justified in Sec. IX) and therefore we ignore the possibility that $2 N^{C} T \gg T_{\mathrm{COH}}$ and $2 N^{D} T \ll T_{\mathrm{COH}}$. Also, it is important to note that the cases $2 N^{C} T \simeq T_{\mathrm{COH}}$ or $2 N^{D} T \simeq T_{\mathrm{COH}}$ (with " $\simeq$ " meaning the same order of magnitude) are undesirable because then the SNR distribution during the computation of $\hat{\lambda}^{C}$ or $\hat{\lambda}^{D}$ (respectively) cannot be predicted (it would not be possible to know what part of the fading distribution we would experience during the computation interval). Fortunately this can always be avoided by choosing a large enough $N^{C}$ or $N^{D}$, hence ensuring that $2 N^{C} T \gg T_{\mathrm{COH}}$ or $2 N^{D} T \gg T_{C O H}$ (though, in general, we are interested in keeping $N^{C}$ and $N^{D}$ as small as possible, since for best performance (i.e., for $U_{M, N^{C}, N^{D}}$ to have unity-gain with as little variance as possible) we strive to generate $\beta_{\chi, d}$ estimates according to the instantaneous SNR $\chi$ and not $\bar{\beta}_{\bar{\chi}, d}$ estimates according to the average SNR $\bar{\chi}$ ).

Other obvious adjustments need to be made to other parameters of Fig. 5 when fading is present. For cases (b) and (c) we must set $\lambda_{\text {Default }}^{D}=\lambda_{\text {Thr }}^{D}=\bar{\beta}_{\bar{\Gamma}, d}$ (where $\bar{\Gamma}$ is the lowest average SNR for which PLL lock is possible in the presence of the fading pdf). For case (c), we must also set $\lambda_{T h r}^{C}=\bar{\beta}_{\bar{\Gamma}, d}$.

It should be noted that since we are talking about a suppressed-carrier M-PSK system, the fact that the system designer has chosen this modulation usually implies that the fading rate is quite slow ([16], [3 p. 250]), so that (in general) cases (a) or (b) would probably apply.

\section{Operation During Tracking}

As noted in Sec. IV, for cases (a) and (b) the operation and performance of $U_{M, N^{C}, N^{D}}$ during tracking is identical to that of $V_{M, N^{C}}$, and this performance is given in [9] where it is shown that $\forall \chi, \beta_{\chi, V}=1$, and thus $\forall \chi, \beta_{\chi, U}=1$. For operation during tracking for case (c), we find that $\hat{\lambda}^{C}$ will give an estimate of $\bar{\beta}_{\bar{\chi}, d}$, and hence $U_{M, N^{C}, N^{D}}$ will have an average effective amplitude suppression factor of unity, that is $\forall \bar{\chi}, \bar{\beta}_{\bar{\chi}, U}=1$. While this is not as good a performance as having $\forall \chi, \beta_{\chi, U}=1$, it will still allow the PLL to maintain optimal parameters, on average (over time), for all $\bar{\chi}$.

\section{Operation DURING ACQUiSITION \& CONTENTS OF LUT D}

As for operation when the carrier is unlocked, all of the components in Fig. 5 have been characterized except for the contents of LUT D, and this is the subject of this section.

As a preliminary step we define the following functions:

$f_{M}^{C}(\chi) \triangleq \beta_{\chi, d}$

$\bar{f}_{M}^{C}(\bar{\chi}) \triangleq \bar{\beta}_{\bar{\chi}, d}=E\left[\beta_{\chi, d} \mid E\left[E_{S} / N_{0}=\bar{\chi}\right]\right]$

$f_{M}^{D}(\chi) \triangleq E\left[\hat{l}_{M, N^{D}}^{D} \mid E_{S} / N_{0}=\chi\right]$

$\bar{f}_{M}^{D}(\bar{\chi}) \triangleq E\left[\hat{l}_{M, N^{D}}^{D} \mid E\left[E_{S} / N_{0}\right]=\bar{\chi}\right]$

where (12) and (14) are computed using (8) (this is discussed further in the appendix). The contents of LUT D, namely the function $\lambda^{\mathrm{D}}=\Psi\left(\hat{l}_{M, N^{D}}^{D}\right)$, are determined according to the cases outlined in Sec. V. We wish to have $\lambda^{\mathrm{D}}$ estimate $\beta_{\chi, d}$ (for case 
(a)), or $\bar{\beta}_{\bar{\chi}, d}$ (for cases (b) or (c)). It is easy to see that for this to happen we must have:

Case (a): $\Psi(x) \triangleq\left(f_{M}^{C} \circ\left(f_{M}^{D}\right)^{-1}\right)(x)$

Cases (b) and (c): $\Psi(x) \triangleq\left(\bar{f}_{M}^{C} \circ\left(\bar{f}_{M}^{D}\right)^{-1}\right)(x)$

In the appendix we discuss methods to evaluate $f_{M}^{C}, \bar{f}_{M}^{C}$, $f_{M}^{D}$ and $\bar{f}_{M}^{D}$. As we show there, these functions are easily determined numerically, from which it follows that determination of $\Psi(x)$ is equally straightforward, and for case (a) this can even be done in closed form as $\Psi(x)=\sqrt{x}$. In Fig. 2 we shown graphs of $\Psi(x)$ which were computed using App. A. As can be seen in Fig. 2, the curve of $\Psi(x)$ is well-behaved, so that only a small dynamic range is necessary to implement LUT D, thus making its implementation quite feasible in fixed-point hardware.

\section{Phase-error Variance Performance}

It can be shown that, due to the fact that $\hat{\lambda}^{C}$ and $\hat{\lambda}^{D}$ change much more slowly than $d_{M}(n)$, the squaring-loss and phase-error variance performance of $U_{M, N^{C}, N^{D}}$ will be identical to that of $d_{M}$, but with the important difference that these results can be achieved with the PLL using $U_{M, N^{C}, N^{D}}$ achieving optimal PLL parameters at all SNRs even when the PLL has a fixed (=nonadaptive) loop filter. This is proven in [9 Secs. 6-8] for operation during tracking and this can similarly be shown for operation during acquisition; here we omit these derivations due to space constraints. Thus, since (as shown in [9]) $d_{M}$ has excellent phaseerror variance performance as compared to other NDA and DD detectors, the same applies to $U_{M, N^{C}, N^{D}}$.

\section{BOUNDS ON $N^{C}$ AND $N^{D}$}

In this section, due to space constraints, we shall assume that the fading is sufficiently slow so that case (a) holds. This is a particularly good assumption for microwave-wavelength satellite communications (see [17]). However, results in the presence of fading are easily achieved using similar procedures, once the fading distribution has been ascertained.

Let us denote the natural frequency and damping factor we are trying to achieve as $\bar{\omega}_{n}$ and $\bar{\zeta}$. We want to achieve them at all $E_{S} / N_{0}=\chi$ in the range $\chi \in[\Gamma, \infty]$ where $\Gamma$ is some reasonable lower bound (e.g., the PLL's lock threshold). In this section we derive expressions for $N^{C}$ and $N^{D}$ necessary to ensure that:

$$
P\left(\left|\omega_{n \chi} / \bar{\omega}_{n}-1\right|<t o l\right)>C, P\left(\left|\zeta_{\chi} / \bar{\zeta}-1\right|<t o l\right)>C
$$

where tol is the acceptable tolerance for $\omega_{n}$ and $\zeta, C$ is the confidence, and $\omega_{n \chi}$ and $\zeta_{\chi}$ are the natural frequency and damping ratio at $E_{S} / N_{0}=\chi$, respectively. For $N^{C}$ in [9 Sec. 9] there it was found that the answer is:

$$
N^{C}>\left(1 / f_{M}^{C}(\Gamma)\right)^{2} \cdot\left(\operatorname{erf}^{-1}(C) / \min \left\{\begin{array}{l}
\left|\left((1-t o l)^{-2}-1\right)\right|, \\
\left|\left((1+t o l)^{-2}-1\right)\right|
\end{array}\right\}\right)^{2}
$$

Following the same sort of derivation in as given in [9 Sec. 9]., we now compute the analogous bounds upon $N^{D}$. It can be easily shown from $[9$ eqs. (26)-(28)] that if we define $\rho \triangleq \sqrt{\beta_{\chi, d} / \Psi\left(\hat{l}_{M, N^{D}}^{D}\right)}$ then we have $\omega_{n \chi}=\rho \bar{\omega}_{n}$ and $\zeta_{\chi}=\rho \bar{\zeta}$. Straightforward manipulations then show that an equivalent constraint to (15) is:
$P\left(\beta_{\chi, d}(1+t o l)^{-2}<\Psi\left(\hat{l}_{M, N^{D}}^{D}\right)<\beta_{\chi, d}(1-t o l)^{-2}\right)>C$.

Since $\Psi(x)$ is monotonically increasing (see Fig. 2), from (17) it is easy to arrive at:

$$
\begin{aligned}
& P\left(\Psi^{-1}\left(\beta_{\chi, d}(1+t o l)^{-2}\right)-E\left[\hat{l}_{M, N^{D}}^{D}\right]<\right. \\
& \left.\hat{l}_{M, N^{D}}^{D}-E\left[\hat{l}_{M, N^{D}}^{D}\right]<\Psi^{-1}\left(\beta_{\chi, d}(1-t o l)^{-2}\right)-E\left[\hat{l}_{M, N^{D}}^{D}\right]\right)>C .
\end{aligned}
$$

Since (see App. A) $E\left[\hat{l}_{M, N^{D}}^{D}\right]=f_{M}^{D}(\chi)=\beta_{\chi, d}^{2}$, then to guarantee

(18) it suffices that:

$P\left(\left|\hat{l}_{M, N^{D}}^{D}-E\left[\hat{l}_{M, N^{D}}^{D}\right]\right|<y^{D}\right)>C$

$$
\text { where } \quad y^{D} \triangleq \min \left\{\left|\Psi^{-1}\left(\beta_{\chi, d}(1-t o l)^{-2}\right)-\beta_{\chi, d}^{2}\right|,\right.
$$

$\left.\left|\Psi^{-1}\left(\beta_{\chi, d}(1+t o l)^{-2}\right)-\beta_{\chi, d}^{2}\right|\right\}$. For case (a) we have (see App. A) $\Psi(x)=\sqrt{x}$ so $\Psi^{-1}(x)=x^{2}$, and we can simplify $y^{D}$ to $y^{D}=\beta_{\chi, d}^{2} \cdot \min \left\{\left|(1-t o l)^{-4}-1\right|,\left|(1+t o l)^{-4}-1\right|\right\}$. Since $\hat{l}_{M, N^{D}}^{D}$ is Gaussian [10 Sec. IV-C] then (19) is equivalent to the constraint

$\operatorname{erf}\left(y^{D} /\left(\sqrt{2} \sqrt{\operatorname{var}\left(\hat{l}_{M, N^{D}}^{D}\right)}\right)\right)>C$

Now, since $\operatorname{var}\left(\hat{l}_{M, N^{D}}^{D}\right) \leq 1.6 /\left(2 N^{D}\right)$ (see [10 Sec. IV-C]), we can solve (20) for $N^{D}$, whereupon we find that for all $E_{S} / N_{0}=\chi \geq \Gamma$ a suitable lower bound on $N^{D}$ would be

$N^{D}>1.6 \cdot\left(\operatorname{erf}^{-1}(C) / y^{D}\right)^{2}$

Fig. 3 shows graphs of $N^{C}$ and $N^{D}$ for various confidences and tolerances. As we can see, only relatively small $N^{C}$ and $N^{D}$ are required in order to ensure good performance above reasonable SNRs for the respective modulations. The thresholds are $E_{S} / N_{0}=-3 \mathrm{~dB}, 0 \mathrm{~dB}, 8 \mathrm{~dB}$, and $13.5 \mathrm{~dB}$ for $M=2,4,8,16$, respectively (these thresholds are a reasonable choice considering practical performance of these modulations; see [5 Sec. III]). The intersection point of the vertical threshold lines with the curves signifies values of $N^{C}$ and $N^{D}$ which will ensure the desired tolerance and confidence at and above the threshold. As we see in Fig. 3, the value of $N^{C}$ that is required is often significantly lower than that of $N^{D}$, given the same target tolerance and confidence.

In a practical hardware implementation, the designer would likely choose $N^{D}$ and $N^{C}$ to be the lowest powers of 2 that satisfy the requirements, thus allowing the PD to be implemented as in Fig. 5 (i.e., to avoid implementation of the divisions by $2 N^{C}$ and $2 N^{D}$, see [9 Sec. 5.2], [10 Sec. III-C]). The right-hand $\mathrm{Y}$-axis of Fig. 3 is in powers-of- 2 units, and aids in this selection.

\section{Conclusions}

In this paper we presented an adaptive phase detector for MPSK carrier PLLs. This phase detector has a constant unity gain both during tracking and acquisition, which allows the PLL to perform optimally at all SNRs. Moreover, the detector was shown to have a compact fixed-point hardware implementation whose performance is robust vis-à-vis AGC circuit imperfections. Operation during fading was also discussed and it was shown that in that case the PLL's parameters remain optimal on average. As for phase-error variance performance, we briefly outlined derivations which show that the proposed detector has excellent phase-error variance performance. Hence, due to the aforementioned characteristics, this detector has immediate applications in FPGA-based or ASICs-based M-PSK receivers

\section{APPENDIX A - ForMUlas FOR $\Psi(x)$}

In this appendix we shall provide formulas for $f_{M}^{C}, \bar{f}_{M}^{C}, f_{M}^{D}$ and $\bar{f}_{M}^{D}$ from which $\Psi(x)$ can be derived. First, we define the 
Rician phase distribution [10 eq. (7)]:

$p_{C}(\theta \mid \chi) \triangleq \frac{e^{-\chi}}{2 \pi}\left[1+\sqrt{2 \chi} \cos \theta \cdot e^{\chi \cdot \cos ^{2} \theta} \cdot \int_{-\infty}^{\cos \theta \sqrt{2 \chi}} e^{-y^{2} / 2} d y\right]$

Then from [9 eq. (22)] we have that

$f_{M}^{C}(\chi)=\beta_{\chi, d}=\int_{-\pi}^{\pi} \cos (M \theta) p_{C}(\theta \mid \chi) \cdot d \theta$

and thus:

$\bar{f}_{M}^{C}(\bar{\chi})=\bar{\beta}_{\bar{\chi}, d}=\int_{0}^{\infty}\left(\int_{-\pi}^{\pi} \cos (M \theta) p_{C}(\theta \mid \chi) \cdot d \theta\right) p_{F}(\chi \mid \bar{\chi}) d \chi$

Furthermore, from [10 eq. (25)]

$f_{M}^{D}(\chi)=\frac{\pi \cdot \chi}{4} \cdot \exp (-\chi)\left[I_{(M-1) / 2}\left(\frac{\chi}{2}\right)+I_{(M+1) / 2}\left(\frac{\chi}{2}\right)\right]^{2}$

where $I_{k}(\bullet)$ is the $k$-th order modified Bessel function of the first kind (see [18 Chap. 24]). Thus, from (14):

$\bar{f}_{M}^{D}(\bar{\chi})=\int_{0}^{\infty} \frac{\pi \cdot \chi}{4} \cdot e^{-\chi}\left[I_{(M-1) / 2}\left(\frac{\chi}{2}\right)+I_{(M+1) / 2}\left(\frac{\chi}{2}\right)\right]^{2} p_{F}(\chi \mid \bar{\chi}) d \chi$

The values of the above functions can be easily computed numerically, and thus so can $\Psi(x)$ as defined in Sec. VII. For cases (b) and (c), the curve of $\Psi(x)$ will depend upon the fading distribution and generally does not have a closed-form representation. However, for case (a), it is noted that it can be shown (using derivations of [19 App. 4A]) that:

$f_{M}^{C}(\chi)=\frac{\sqrt{\pi \cdot \chi}}{2} \cdot \exp \left(\frac{-\chi}{2}\right)\left[I_{(M-1) / 2}\left(\frac{\chi}{2}\right)+I_{(M+1) / 2}\left(\frac{\chi}{2}\right)\right]$

which reveals, using (25), that $f_{M}^{D}(\chi)=\left(f_{M}^{C}(\chi)\right)^{2}=\beta_{\chi, d}^{2}$, and it is easy to then show that $\Psi(x) \triangleq\left(f_{M}^{C} \circ\left(f_{M}^{D}\right)^{-1}\right)(x)$ can be expressed in closed form very simply as $\Psi(x)=\sqrt{x}$.

\section{REFERENCES}

[1] J. G. Proakis, Digital communications, 4th ed. Boston: McGraw-Hill, 2001.

[2] H. Meyr, M. Moeneclaey, and S. Fechtel, Digital communication receivers: synchronization, channel estimation, and signal processing. NY: Wiley, 1998

[3] U. Mengali and A. N. D'Andrea, Synchronization techniques for digital receivers. NY: Plenum Press, 1997.

[4] W. C. Lindsey and M. K. Simon, Telecommunication systems engineering. NJ: Prentice-Hall, 1973.

[5] B. T. Kopp and W. P. Osborne, "Phase jitter in MPSK carrier tracking loops: analytical, simulation and laboratory results," IEEE Trans. Commun., vol. 45, no. 11, pp. 1385-1388, Nov. 1997.

[6] W. P. Osborne and B. T. Kopp, "An analysis of carrier phase jitter in an M-PSK receiver utilizing MAP estimation," in Proc. MILCOM '93, Boston, MA, USA, 1993, pp. 465-470.

[7] W. P. Osborne and B. T. Kopp, "Synchronization in M-PSK modems," in Proc. ICC '92, Chicago, IL, USA, 1992, pp. 1436-1440.

[8] R. De Gaudenzi, T. Garde, and V. Vanghi, "Performance analysis of decision-directed maximum-likelihood phase estimators for M-PSK modulated signals," IEEE Trans. Commun., vol. 43, no. 12, pp. 3090-3100, Dec. 1995.

[9] Y. Linn, "A Robust Phase Detection Structure for M-PSK: Theoretical Derivations, Simulation Results, and System Identification Analysis," in Proc. 18th Canadian Conference on Electrical and Computer Engineering (CCECE'05), Saskatoon, Saskatchewan, Canada, May 1-4, 2005, pp. 869-883.

[10] Y. Linn, "A Real-Time SNR Estimator for D-MPSK over Frequency-Flat Slow Fading AWGN Channels," in Proc. 2006 IEEE Sarnoff Symposium, Princeton, NJ, Mar. 27-28, 2006.

[11] F. M. Gardner, Phaselock techniques, 2nd ed. NY: Wiley, 1979.

[12] A. Blanchard, Phase-locked loops. Application to coherent receiver design. NY: Wiley, 1976.

[13] Y. Linn and N. Peleg, "A family of self-normalizing carrier lock detectors and Es/N0 estimators for M-PSK and other phase modulation schemes," IEEE Trans. Wireless Commun., vol. 3, no. 5, pp. 1659-1668, Sep. 2004.

[14] Y. Linn, "Quantitative analysis of a new method for real-time generation of SNR estimates for digital phase modulation signals," IEEE Trans. Wireless Commun., vol. 3, no. 6, pp. 1984-1988, Nov. 2004.
[15] M. K. Simon and M. Alouini, "A unified approach to the performance analysis of digital communication over generalized fading channels," Proc. IEEE, vol. 86, no. 9, pp. 1860-1877, Sep. 1998

[16] B. Sklar, "Rayleigh Fading Channels in Mobile Digital Communication Systems Part II: Mitigation," IEEE Comm. Mag., vol. 35, no. 9, pp. 148-155, Sep. 1997.

[17] M. M. J. L. van de Kamp, "Climatic radiowave propagation models for the design of satellite communication systems," $\mathrm{PhD}$. Thesis, Technische Universiteit Eindhoven, The Netherlands, 1999.

[18] M. R. Spiegel, Mathematical handbook of formulas and tables. NY: McGraw-Hill, 1968.

[19] K.-P. Ho, Phase-modulated optical communication systems. NY: Springer, 2005.

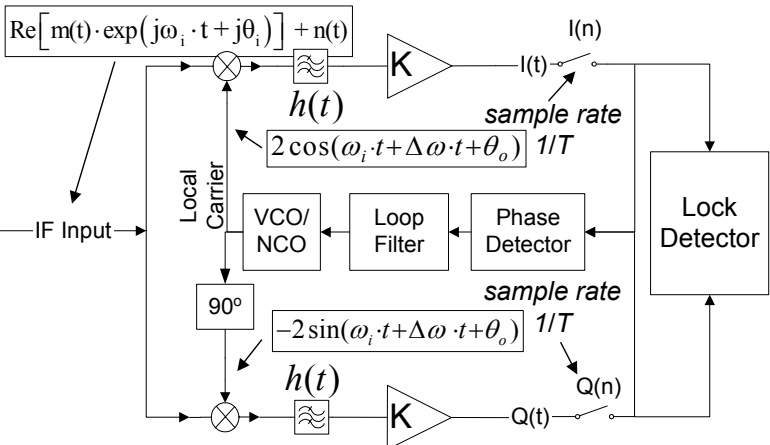

Fig. 1 - Simplified M-PSK receiver model.

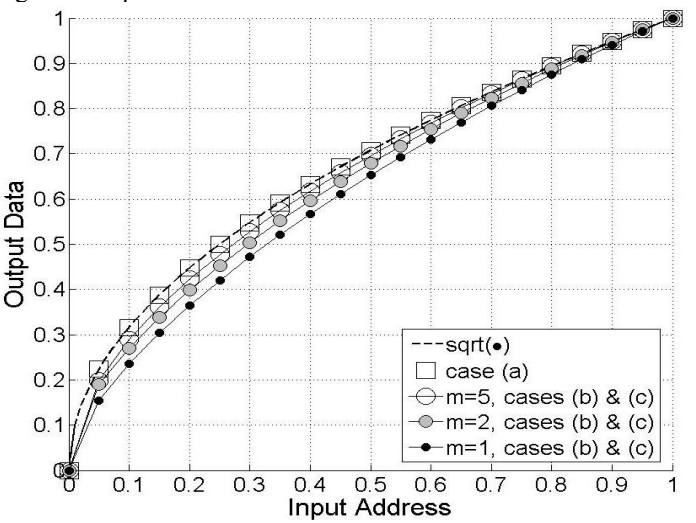

Fig. 2 - Contents of LUT D (calculated via App. A) which implements the function $\Psi(x)$ for $M=4$ (QPSK). For case (a), we see that indeed

$\Psi(x)=\sqrt{x}$. For cases (b) and (c), Nakagami- $m$ fading is assumed (i.e. the fading pdf of (9)) and results are given for different values of the $m$ parameter. For convenience, the input address and the output data of the LUT are assumed to represent fixed-point numbers between 0 and 1 .

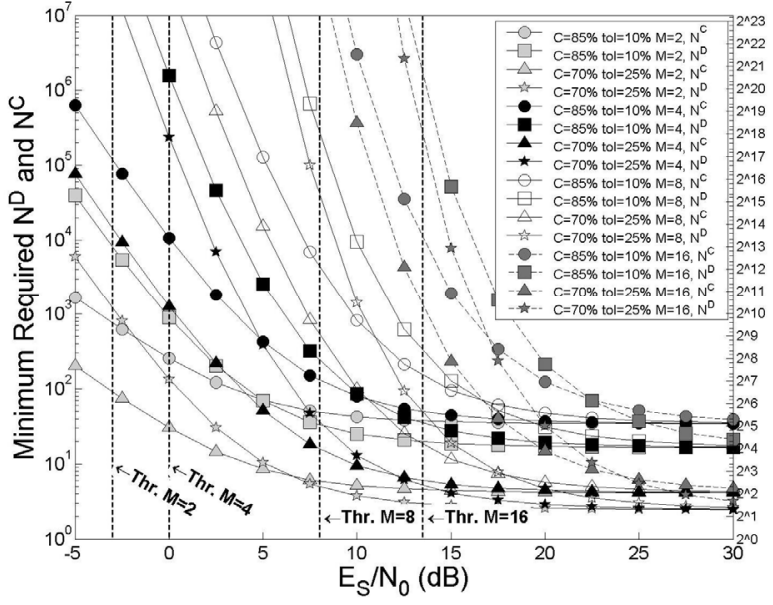

Fig. 3 - Minimum required $N^{D}$ and $N^{C}$ for case (a). The vertical dashed lines show the PLL lock thresholds for the respective modulations. 


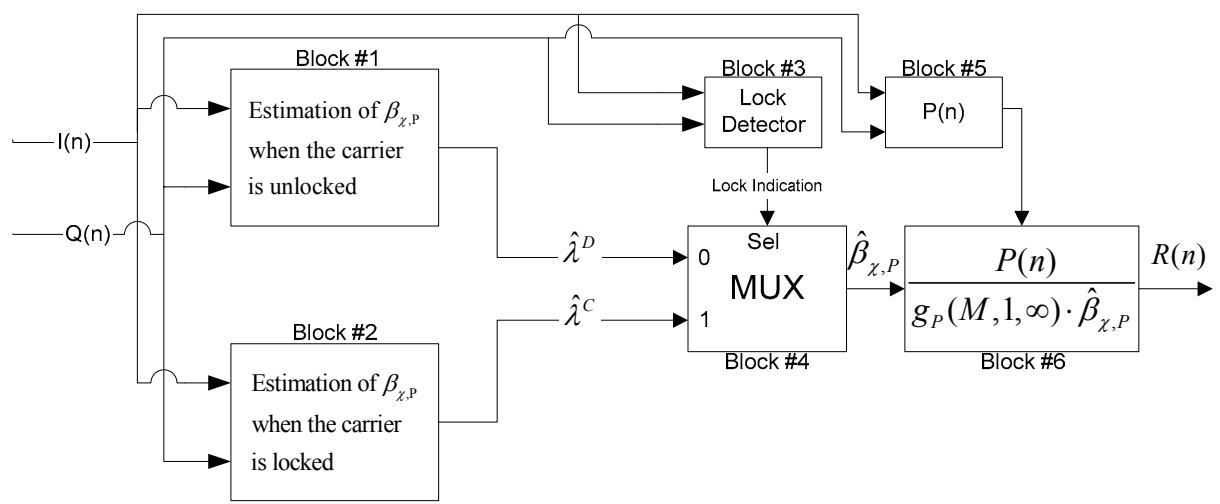

Fig. 4 - Top-level schematic of methodology for generating a constant-gain detector $R(n)$ from a given phase detector $P(n)$. The general idea is to estimate $\beta_{\chi, P}$ (this estimate is denoted as $\hat{\beta}_{\chi, P}$ ) and divide $P(n)$ by $g_{P}(M, 1, \infty) \cdot \hat{\beta}_{\chi, P}$ so that $\forall \chi, \beta_{\chi, R}=1$.

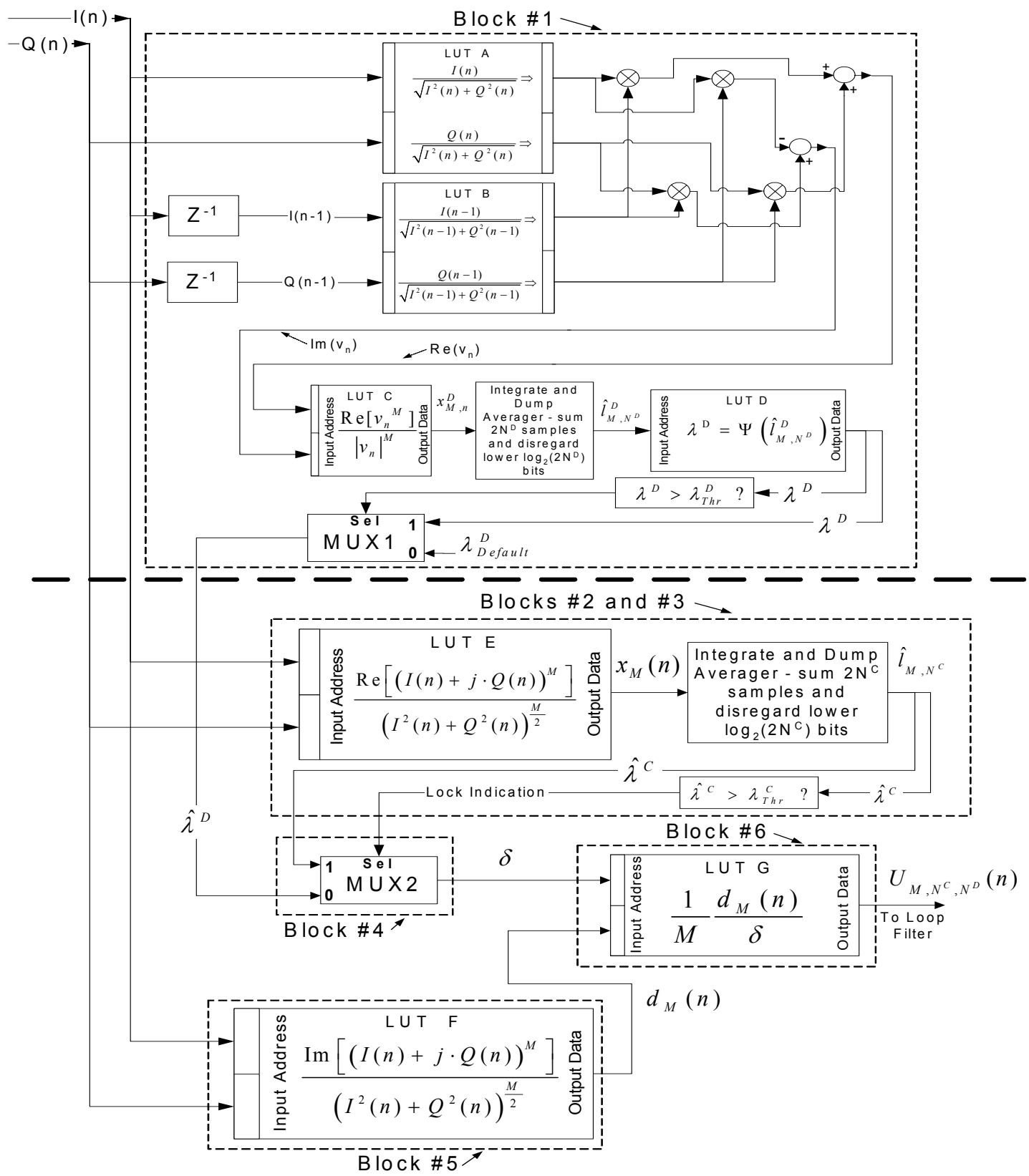

Fig. 5 - Fixed-point hardware implementation of $U_{M, N^{C}, N^{D}}$. LUT signifies Lookup Table, i.e. a ROM (Read-Only Memory) whose output is determined as a function of its input address. Note that in all the datapaths, some lower-order bits may be discarded in order to reduce the size of the lookup tables (see example in [10 Fig. 2], and discussion in [10 Secs. III-C, IV-A]); this is not shown in this figure for simplicity. 